\title{
Day 4 embryos should not be underestimated in IVF.
}

\author{
Noora Kaartinen ${ }^{1 *}$, Kirsi Kananen², Candido Tomás², Helena Tinkanen ${ }^{1}$ \\ ${ }^{1}$ Department of Obstetrics and Gynaecology, Tampere University Hospital, Teiskontie 35, Tampere, Finland \\ ${ }^{2}$ Fertility Clinic Ovumia, Biokatu 12, 33520 Tampere
}

\begin{abstract}
Background: 2-3 and Day 5 fresh and frozen-thawed embryos are most frequently used in ART, while the results of Day 4 embryo transfers are seldom reported in the literature. However, the use of Day 4 embryos in clinical practice increases flexibility when choosing the embryo transfer day, and enables avoiding embryo transfers on weekends.

Methods and findings: We conducted a retrospective cohort study (1.1.2009-31.12.2014) comparing the clinical outcomes of fresh Day $3(n=1371)$, Day $4(n=360)$ and Day $5(n=326)$ and vitrified-warmed Day $3(n=635)$, Day $4(n=175)$ and Day $5(n=654)$ embryos. The live birth rates of fresh Day 3, Day 4 and Day 5 embryo transfers were $26.5 \%, 31.4 \%$ and $24.5 \%$, respectively $(p=0.026)$. The miscarriage rates were $16.6 \%, 11.7 \%$ and $23.0 \%$, respectively $(p=0.017)$. The live birth rates of Day 3, Day 4 and Day 5 vitrified-warmed embryo transfers were $22.0 \%$, $30.9 \%$ and $22.6 \%$, respectively $(p=0.024)$. The miscarriage rates were $21.6 \%, 15.9 \%$ and $27.8 \%$, respectively $(\mathbf{p}=0.049)$.
\end{abstract}

Conclusions: The results of the present study show that Day 4 embryo transfers produce good clinical outcomes and can be considered for wider utilization at IVF clinics.

Keywords: Embryo transfer, Retrospective, Vitrified, Birth rates, Miscarriage, IVF.

Accepted on December 27, 2017

\section{Introduction}

In IVF, the routine of replacing Day 2-3 embryos into the uterus started to change due to the development of the sequential media, enabling the embryo culture to the blastocyst stage. The opportunity to transfer embryos at the blastocyst stage generated expectations of superior clinical outcomes which were based on the rationale of better embryo-endometrial synchrony [1]. The further advantage of the blastocyst culture was considered to be the improved embryo selection during the extended embryo culture [2]. Due to the potential embryo wastage associated with the blastocyst culture, only patients with a sufficient number of embryos at the cleavage stage are suitable candidates for a blastocyst culture.

Embryo compaction at the morula stage, approximately on culture day 4 , is the pre-requisite for blastocoel formation and blastocyst development. The compaction can be detected as a gradual disappearance of clear cellular borders and a tightening of cellular contacts. In practice it is difficult to count the exact cell number of a compacted morula. As the compaction gradually proceeds, the outer cells of the embryo form tight junctions between each other and the blastocoel starts to form in the middle of the embryo.

The rationale for transferring Day 4 embryos is similar to Day 5 embryos: the preferable endometrium-embryo synchrony as well as the in vitro selection of embryos capable of developing through critical steps under the control of its own genome. The Day 4 embryo transfer was first reported by Huisman [3]. Thereafter a few studies of Day 4 embryo transfers have been published. In a retrospective study, Tao et al. detected a higher implantation rate despite a lower number of fresh embryos transferred on Day 4 compared to Day 3 [4]. In a study by Pantos et al., the protocol of fresh Day 4 transfers was associated with a trend towards a higher clinical pregnancy rate without statistical significance in comparison with Day 3 fresh transfers [5]. However, Kang et al. did not detect a difference in the pregnancy rates between Day 4 and Day 5 fresh single embryo transfers [6]. In Lee's study, a lower implantation rate, yet a similar clinical pregnancy rate were detected after Day 4 fresh embryo transfers compared to Day 5 fresh embryo transfers [7]. Deidre Zander-Fox compared the two cryopreservation methods: slow freezing and vitrification of mouse morulae and early blastocysts. Vitrification resulted in the better survival of morulae and early blastocysts as well as a higher rate of blastocyst development compared to slow freezing [8].

Day 4 embryo transfers have been used at our clinic for several years. To survey the effect of the utilization of Day 4 embryos on the pregnancy results, we conducted a retrospective study to examine the clinical outcomes of fresh and vitrified-warmed Day 4 embryo transfers compared to Day 3 and Day 5 fresh and vitrified-warmed embryo transfers.

\section{Materials and Methods}

The study was given permission to proceed by the institutional review board of Tampere University Hospital on the $6^{\text {th }}$ October 2015. The study material was retrospectively collected from the patient records from the time period of 1.1.2009-31.12.2014. All fresh Day 3, Day 4 and Day 5 embryo transfer cycles were collected as well as vitrified-warmed Day 3, Day 4 and Day 5 embryo transfers. The Day 3 and Day 4 embryos were cultured overnight after thawing and transferred on the next day. The Day 5 transfers were performed on the day of warming. 
The controlled ovarian hyperstimulation protocol: The controlled ovarian hyperstimulation was accomplished using an antagonist, long agonist or short agonist protocol. Recombinant FSH was used to induce follicular growth and the hCG was administered when the leading follicle reached $18 \mathrm{~mm}$ in the agonist protocol and $17 \mathrm{~mm}$ in the antagonist protocol. Thirtysix hours after the hCG injection, the oocytes were collected using a transvaginal ultrasound-guided puncture. The luteal support consisted of vaginally administered progesterone and the embryo transfer was made under ultrasound monitoring.

Culture conditions: The culture media was supplied by Origio (MålØv, Denmark) or Vitrolife (Gothenburg, Sweden). Successful fertilization was confirmed 16 to 18 hours after the insemination (hpi). The normally fertilized zygotes were cultured in sequential media using ISM1 ${ }^{\mathrm{TM}}$ (Origio) until day 2 when they were moved to culture in BlastAssist TM (Origio) until day 3, 4 or 5, or in G-1 ${ }^{\mathrm{TM}}$ PLUS (Vitrolife) until day 3 when they were moved to either BlastAssist ${ }^{\mathrm{TM}}$ (Origio) or G-2 ${ }^{\mathrm{TM}}$ PLUS (Vitrolife) to culture until day 4 or 5 . The culture in the blastocyst media was performed in reduced oxygen $(6 \%$ $\left.\mathrm{CO}_{2} / 6 \% \mathrm{O}_{2} / 88 \mathrm{~N}_{2}\right)$.

Embryo evaluation and selection: The duration of the embryo culture and the date of the fresh embryo transfer were determined based on the number of retrieved oocytes, the patients' treatment history and the weekday of the oocyte pickup. If the number of oocytes was less than 10, there had been a low fertilization rate in the previous treatments, or the oocyte pick-up day was Monday or Tuesday, a shorter culture period (3-4 days) was chosen. If the number of oocytes exceeded ten or the oocyte pick-up day was Thursday or Friday, a longer culture period (4-5 days) was chosen.
The embryos were evaluated on the day of the fresh embryo transfer. The evaluation of the Day 3 embryos was performed by applying a modified scoring system $[9,10]$. The expected developmental stage (i.e. four cells on Day 2 or eight cells on Day 3 ) and the presence of more than $10 \%$ fragmentation; an unequal size of blastomeres; a large perivitelline space; cytoplasmic abnormalities or multinucleated blastomeres were used to score the embryos. The Day 4 embryos were evaluated according to the criteria explained in Table 1 and Figures 1-6. The Day 5 embryos were evaluated according to Gardner and Schoolcraft. An embryo was selected for cryopreservation by vitrification if it had reached at least a fully compacted morula stage by Day 5 or the true blastocyst stage (i.e. stage 3 ) by Day 6 [11]. At the blastocyst stages 2-6, the inner cell mass and trofectoderm class A or B had to be present. One or two of the best quality embryos were selected for fresh embryo transfer on Day 3, 4 or 5. The remaining top or good quality embryos were selected for cryopreservation by vitrification. Poor quality embryos were cultured further and vitrified on Day 5 or Day 6 if they developed into good quality blastocysts.

Cryopreservation and warming protocols: The vitrification and warming were performed according to the manufacturer's instructions (VitriFreezeES ${ }^{\text {TM }}$ VitriThawES ${ }^{\mathrm{TM}}$, FertiPro, Beernem, Belgium). High security vitrification straws (Cryo BioSystems, L'aigle, France) were used as the vitrification device.

The Day 3 and Day 4 embryos were warmed one day before the embryo transfer and the survived embryos (minimum $50 \%$ of blastomeres alive) were cultured overnight in Blast Assist.

Table 1: The embryo quality classification of Day 4 embryos.

\begin{tabular}{|l|l|}
\hline Embryo quality & Description \\
\hline Top quality & $\begin{array}{l}\text { Appearance of early cavity or a high rate of compaction }(75-100 \%) \text { as well as a large total embryonic mass (minimum } 75 \% \text { of putative } \\
\text { embryonic cells forming the embryo). }\end{array}$ \\
\hline Good quality & $\begin{array}{l}\text { Appearance of early cavity with a medium sized embryonic mass (approx. } 50 \% \text { of putative embryonic cells), or a low rate of compaction } \\
\text { (approx. } 50 \%) \text { with a big total embryonic mass (see above), or a high rate of compaction with a medium sized total embryonic mass, or } \\
\text { pre-compaction morula stage embryo with } \geq 12 \text { cells and a low fragmentation index. }\end{array}$ \\
\hline Poor quality & $\begin{array}{l}\text { Appearance of early cavity with a small embryonic mass ( }<50 \% \text { of putative embryonic cells), or from a medium to high rate of compaction } \\
\text { with a small embryonic mass, or a pre-compaction morula stage embryo with }<12 \text { cells and a high fragmentation index. }\end{array}$ \\
\hline
\end{tabular}

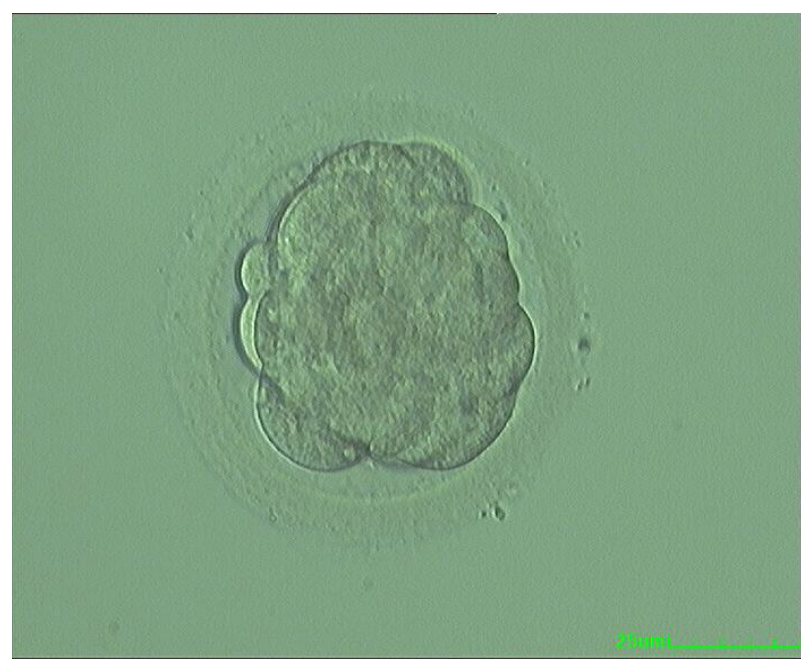

Figure 1: The embryos were at the morula stage.

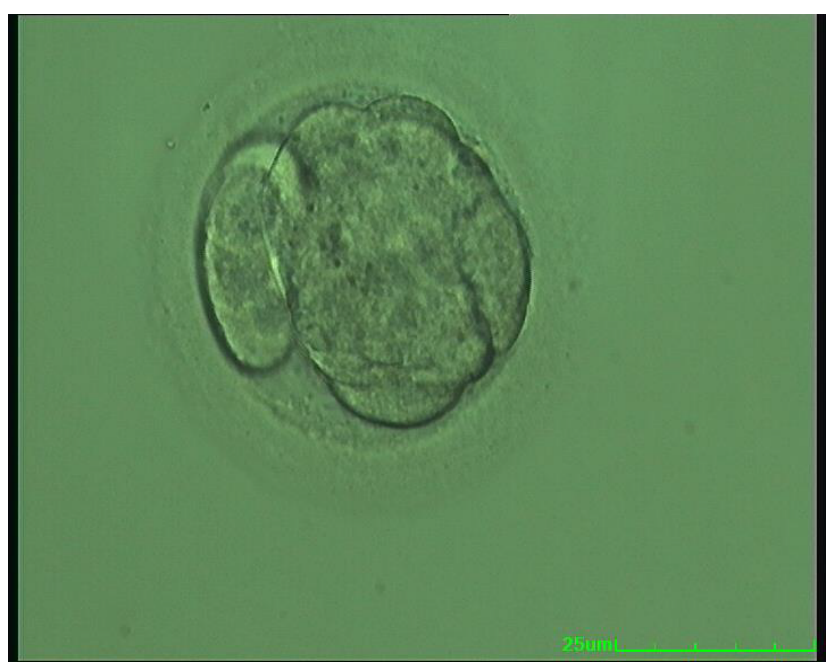

Figure 2: The embryos were at the compaction stage. 


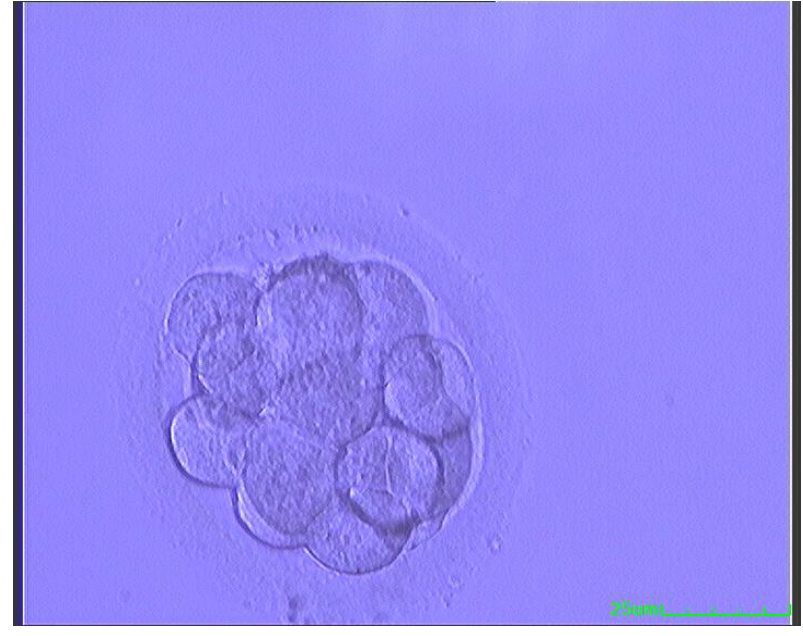

Figure 3: The embryos were at the early blastocyst stage.

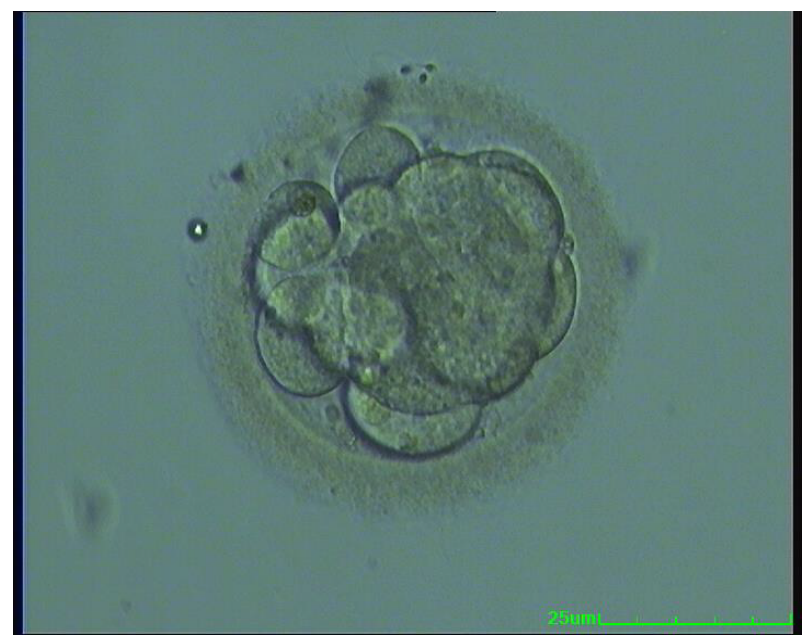

Figure 4: Low rate of compaction (approx. 50\%) with a big total embryonic mass.

The embryos that had cleaved were evaluated and selected for embryo transfer ( 1 or 2 embryos). The Day 5 embryos were warmed on the day of the embryo transfer. Embryo survival was defined as cleavage during an over-night culture (Day 3 and 4 embryos) or the survival of minimum $50 \%$ of embryonic cells (Day 5 embryos), respectively.

Vitrified-warmed embryo transfers: The vitrified-warmed embryo transfers (FET) were performed in spontaneous or hormone-substituted cycles. In hormone-substituted cycles, estradiol hemihydrate as tablets ( $2 \mathrm{mg}$ three times a day) or transdermal patches (100-150 $\mu \mathrm{g}$ twice a week) was used from the beginning of the menstrual cycle, in addition to natural vaginal progesterone tablets $(200 \mathrm{mg} \times 3$ a day), starting when the endometrial thickness measured 7-8 $\mathrm{mm}$. One or two of the embryos were transferred using ultrasound guidance.

Pregnancy monitoring: Clinical pregnancy was assessed with a transvaginal ultrasound 5 weeks after the embryo transfer and defined as the presence of at least one gestational sac on the ultrasound scan. A spontaneous abortion was considered to

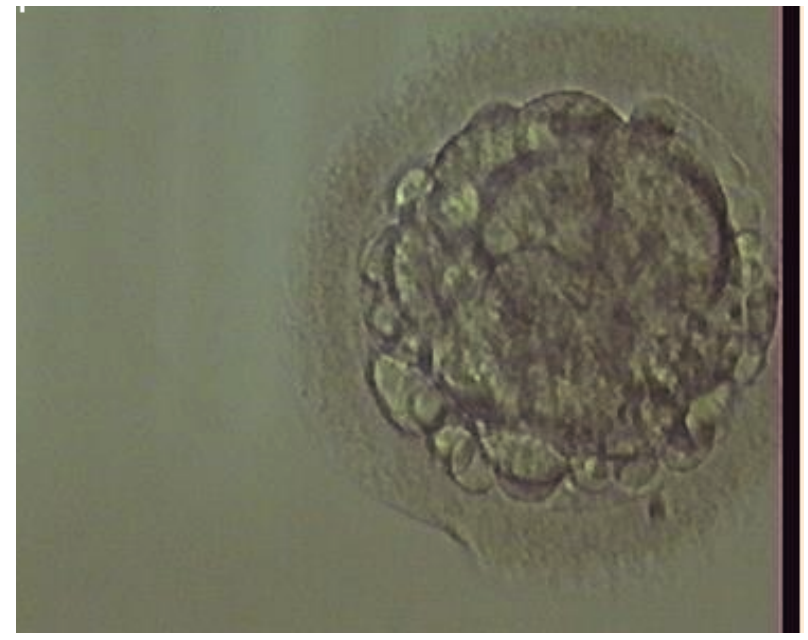

Figure 5: High rate of compaction with a medium sized total embryonic mass.

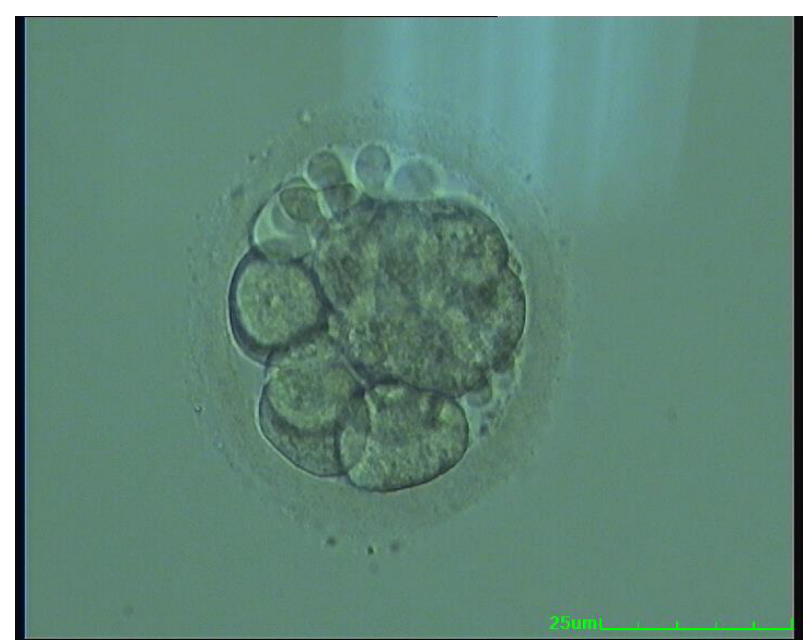

Figure 6: Pre-compaction morula stage embryo with $<12$ cells and a high fragmentation index.

be a pregnancy loss until week 22 of gestation after a previous confirmation of a clinical pregnancy.

Statistical analyses: The Chi-square test was used to compare the delivery rates, clinical pregnancy rates and miscarriage rates between the groups. The Kruskal-Wallis test was used for the statistical comparison of the cryo-survival rates and the number of oocytes and vitrified embryos. For a comparison between culture days, the Bonferroni-adjusted p-values are presented. All the statistical analyses were performed using the IBM SPSS software (v19.0 Armonk, NY, USA).

\section{Results}

During the study period, we performed 1371 Day 3; 360 Day 4; and 326 Day 5 fresh embryo transfers and 635 Day 3; 175 Day 4; and 654 Day 5 vitrified-warmed embryo transfers. The mean age, mean BMI, and total FSH dose were similar across all the groups. The duration of infertility and the proportion of cycles stimulated with an antagonist protocol were different between the fresh Day 4 and Day 5 transfers (Table 2). All the Day 5 embryos were transferred on the day of warming, whereas the Day 3 and Day 4 embryos were transferred on the next day after 
Citation: Kaartinen N, Kananen K, Tomás C, et al. Day 4 embryos should not be underestimated in IVF. Gynecol Reproduct Endocrinol-UK. 2017;1(1):35-41

warming. In fresh IVF/ICSI cycles the mean number of frozen embryos was the lowest in the Day 4 group and highest in the Day 5 group. After fresh Day 4 embryo transfers the live birth rate was higher and miscarriage rate lower compared to Day 3 or Day 5 transfers, yet the clinical pregnancy rates were similar (Table 3).

The clinical pregnancy rate of the vitrified-warmed Day 4 embryos was significantly higher than the Day 3 vitrifiedwarmed embryos and similar to the Day 5 vitrified-warmed embryos (Table 4). The Day 4 vitrified-warmed embryos resulted in a significantly higher live birth rate than the Day 3 or Day 5 vitrified-warmed embryos. The mean number of embryos transferred in the vitrified-warmed cycles for the
Day 3, Day 4 and Day 5 embryos were 1.21, 1.20, and 1.19, respectively.

To further study the effect of the embryo development rate on the clinical outcomes, the fresh Day 4 embryos were allocated to three subgroups based on their developmental stage at transfer. In Group 1, the embryos were at the morula stage (Figure 1), in Group 2 at the compaction stage (Figure 2), and in Group 3 at the early blastocyst stage (Figure 3). The live birth rate was the highest and the miscarriage rate the lowest in the early blastocyst group (Group 3) (47.4\% and 6.7\% respectively), while at the morula stage (Group 1), the live birth rate was the lowest and the miscarriage rate the highest $(19.4 \%$ and $15.4 \%$, respectively) (Table 5).

Table 2: Patient characteristics of fresh Day 3, Day 4 and Day 5 embryo transfer (ET) cycles.

\begin{tabular}{|c|c|c|c|c|c|c|c|c|}
\hline & \multicolumn{2}{|c|}{$\begin{array}{l}\text { Fresh } \\
\text { Day } 3\end{array}$} & \multirow{2}{*}{$\begin{array}{c}\text { p-value } \\
\text { Day } 3 \text { vs. Day } 4\end{array}$} & \multicolumn{2}{|c|}{$\begin{array}{l}\text { Fresh } \\
\text { Day } 4\end{array}$} & \multicolumn{2}{|c|}{$\begin{array}{l}\text { Fresh } \\
\text { Day } 5\end{array}$} & \multirow{2}{*}{$\begin{array}{c}\text { p-value } \\
\text { Day } 4 \text { vs. Day } 5\end{array}$} \\
\hline & $\mathbf{n}$ & $\%$ & & $\mathbf{n}$ & $\%$ & $\mathbf{n}$ & $\%$ & \\
\hline Embryo transfers & 1371 & & & 360 & & 326 & & \\
\hline $\begin{array}{l}\text { Mean number of embryos / } \\
\text { transfer }\end{array}$ & 1.25 & & & 1.21 & & 1.14 & & \\
\hline Age (Years) & $32.2 \pm 4.2$ & & 0.451 & $32.0 \pm 4.1$ & & $31.6 \pm 4.1$ & & 0.599 \\
\hline BMI $\left(\mathrm{kg} / \mathrm{m}^{2}\right)$ & $24.7 \pm 4.5$ & & 0.689 & $24.6 \pm 4.3$ & & $24.4 \pm 3.9$ & & 0.15 \\
\hline Duration of infertility (Years) & 3.21 & & 0.917 & 3.26 & & 3 & & 0.003 \\
\hline ICSI & 708 & 51.6 & 0.517 & 178 & 49.4 & 175 & 53.7 & 0.268 \\
\hline Primary infertility & 787 & 57.4 & 0.68 & 211 & 58.6 & 167 & 51.2 & 0.052 \\
\hline Antagonist & 800 & 58.4 & 0.582 & 216 & 60.0 & 147 & 45.1 & $<0.001$ \\
\hline Total FSH dose (IU) & 1941 & & 0.428 & 1860 & & 1835 & & 0.744 \\
\hline The mean number of oocytes & 11 & & $<0.001^{*}$ & 12.8 & & 16.3 & & $<0.001^{*}$ \\
\hline $\begin{array}{c}\text { The mean number of vitrified } \\
\text { embryos }\end{array}$ & 2.37 & & $0.711^{\star}$ & 2.13 & & 3.18 & & $<0.001^{*}$ \\
\hline
\end{tabular}

Table 3: The clinical outcomes of fresh Day 3, Day 4 and Day 5 embryo transfers. P-values for comparisons with Day 4 outcomes.

\begin{tabular}{|c|c|c|c|c|c|c|c|c|}
\hline & \multicolumn{2}{|c|}{ Day 3} & \multirow{2}{*}{ p-value Day 3 vs. Day 4} & \multicolumn{2}{|c|}{ Day 4} & \multicolumn{2}{|c|}{ Day5 } & \multirow{2}{*}{$\begin{array}{c}\text { p-value Day } 3 \text { vs. } \\
\text { Day } 5\end{array}$} \\
\hline & $\mathbf{n}$ & $\%$ & & $\mathbf{n}$ & $\%$ & $\mathbf{n}$ & $\%$ & \\
\hline Embryo transfers & 1371 & & & 360 & & 326 & & \\
\hline $\begin{array}{c}\text { Mean number of embryos per } \\
\text { transfer }\end{array}$ & 1.25 & & 0.384 & 1.21 & & 1.14 & & 0.085 \\
\hline CPR & 465 & 33.9 & 0.142 & 137 & 38.1 & 113 & 34.7 & 0.356 \\
\hline LBR & 364 & 26.5 & 0.032 & 116 & 31.4 & 80 & 24.5 & 0.026 \\
\hline MCR & 77 & 16.6 & 0.165 & 16 & 11.7 & 26 & 23 & 0.017 \\
\hline
\end{tabular}

Table 4: The clinical outcomes of Day 3, Day 4 and Day 5 vitrified-warmed embryo transfers.

\begin{tabular}{|c|c|c|c|c|c|c|c|c|}
\hline & \multicolumn{2}{|c|}{$\begin{array}{l}\text { Warmed } \\
\text { Day } 3\end{array}$} & \multirow{2}{*}{$\begin{array}{c}\text { p-value } \\
\text { Day } 3 \text { vs. Day } 4\end{array}$} & \multicolumn{2}{|c|}{$\begin{array}{l}\text { Warmed } \\
\text { Day } 4\end{array}$} & \multicolumn{2}{|c|}{$\begin{array}{l}\text { Warmed } \\
\text { Day } 5\end{array}$} & \multirow{2}{*}{$\begin{array}{c}\text { p-value } \\
\text { Day } 4 \text { vs. Day } 5\end{array}$} \\
\hline & $\mathbf{n}$ & $\%$ & & $\mathbf{n}$ & $\%$ & $\mathbf{n}$ & $\%$ & \\
\hline Embryo transfers & 635 & & & 175 & & 654 & & \\
\hline Mean number of embryos transferred & 1.21 & & 1 & 1.2 & & 1.19 & & 1 \\
\hline Survival rate & 0.93 & & $0.031^{*}$ & 0.89 & & $0.90^{*}$ & & 1 \\
\hline CPR & 190 & $(29.9)$ & 0.017 & 69 & $(39.4)$ & 209 & $(32.0)$ & 0.63 \\
\hline LBR & 140 & $(22.0)$ & 0.016 & 54 & $(30.9)$ & 148 & $(22.6)$ & 0.024 \\
\hline MCR & 41 & $(21.6)$ & 0.317 & 11 & $(15.9)$ & 58 & $(27.8)$ & 0.049 \\
\hline
\end{tabular}


Table 5: The clinical outcome of fresh top and good quality Day 4 embryos divided in 3 groups according to the developmental stage. In Group 1 the embryos are at the morula stage, before compaction. In the Group 2 the embryos are in the compaction process and in the Group 3 the embryos are at the early blastocyst stage.

\begin{tabular}{|c|c|c|c|c|c|c|c|c|c|}
\hline & \multicolumn{2}{|c|}{$\begin{array}{c}\text { Group } 1 \\
\text { Morula stage, before } \\
\text { compaction }\end{array}$} & \multirow[t]{2}{*}{$\begin{array}{l}\text { p-value } \\
\text { Group1 } \\
\text { vs. } \\
\text { Group3 }\end{array}$} & \multicolumn{2}{|c|}{$\begin{array}{c}\text { Group } 2 \\
\text { Compacted morula }\end{array}$} & \multirow[t]{2}{*}{$\begin{array}{l}\text { p-value } \\
\text { Group } 1 \\
\text { Vs. } \\
\text { Group } 2\end{array}$} & \multicolumn{2}{|c|}{$\begin{array}{c}\text { Group } 3 \\
\text { Early blastocyst }\end{array}$} & \multirow[t]{2}{*}{$\begin{array}{c}\text { p-value } \\
\text { Group } 2 \\
\text { vs. } \\
\text { Group } 3\end{array}$} \\
\hline & $\mathbf{n}$ & $\%$ & & $\mathbf{n}$ & $\%$ & & $n$ & $\%$ & \\
\hline $\begin{array}{l}\text { Number of embryo } \\
\text { transfers }\end{array}$ & 103 & & & & 200 & & & 57 & \\
\hline CPR & 26 & 25.2 & $<0.001$ & 81 & 40.5 & 0.008 & 30 & 52.6 & 0.103 \\
\hline LBR & 20 & 19.4 & $<0.001$ & 69 & 34.5 & 0.006 & 27 & 47.4 & 0.076 \\
\hline MCR & 4 & 15.4 & 0.401 & 10 & 12.3 & 0.741 & 2 & 6.7 & 0.508 \\
\hline
\end{tabular}

\section{Discussion}

The main finding of the present study is the superior clinical outcome of fresh and vitrified-thawed Day 4 transfers in comparison with embryos cultured for 3 or 5 days. In particular, the pregnancy rates of early blastocysts and the compacted morulae on culture day 4 produced a high clinical pregnancy rate and live birth rate and a low miscarriage rate. Clinically, the differences appeared notable and encouraged the practice of vitrifying the embryos after four days of culture and transferring them after an overnight culture.

The literature addressing the length of the embryo culture before a fresh transfer or cryopreservation in IVF/ICSI treatments commonly debate the benefits of a culture lasting until the cleavage stage (Day 2-3) or the blastocyst stage (Day 5-6). Traditionally, the benefits of the blastocyst culture have been considered to be the higher implantation potential, probably due to the natural selection of embryos during the extended culture. The disadvantage of the blastocyst culture is the loss of embryos during the culture and the risk of having no embryos for transfer after five days of culture. According to the scientific literature, the use of Day 4 embryos has been fairly uncommon. One of the reasons could be the difficulty of assessing the embryo quality. In theory, the transfer of Day 4 embryos combines the advantages of the cleavage stage embryo transfers; the activation of the embryonic genome has already occurred, and the time period of embryos being exposed to an unnatural environment is shorter than in the blastocyst culture. During its first days of existence, the embryo undergoes massive epigenetic reprogramming. When it is predisposed to an in vitro culture during this delicate period, the risk of epigenetic changes increases.

According to the results, the delivery rate was the highest and the miscarriage rate the lowest after fresh Day 4 embryo transfers compared to Day 3 and Day 5 transfers. The retrospective design of the study rouses the question of whether the patients with a good prognosis are overrepresented in the Day 4 embryo transfer group. The background of the patients, their mean age, mean BMI and mean total FSH dose were all similar between the groups, which could indicate that the patient material was rather similar across the groups [12].

The difference between the number of oocytes collected from the Day 3 and Day 4 fresh embryo transfer groups was small, albeit statistically significant. With a similar mean age, BMI and FSH dose a significant difference in the patient material between groups is unlikely, yet cannot be ruled out. However, there were more oocytes collected in the Day 5 transfer group, compared to the Day 4 transfer group (16.3 oocytes vs. 12.8 oocytes), which could be interpreted as a sign of a higher percentage of goodprognosis patients in the Day 5 embryo transfer group. It also reflects the tendency of extending the culture length based on the number of oocytes collected. Nevertheless, the results of the Day 4 embryo transfers were superior to the Day 5 embryo transfers. In a retrospective study by Holschbach (2017), comparable clinical pregnancy rates after Day 4 and Day 5 embryos were detected [13]. The miscarriage rates or live birth rates were not reported. In our study, the clinical pregnancy rates of fresh Day 4 and Day 5 were similar, yet the live birth rate of the Day 4 embryos was statistically significantly higher and miscarriage rate lower than the Day 5 transfers.

At our clinic, no embryo transfers were performed on weekends. Therefore the weekday of the oocyte pick-up had an important effect on the culture length. If the oocyte pick-up was undertaken on a Monday, the embryos could not be cultured to the blastocyst stage due to the weekend, and for the Tuesday pick-ups the longest possible embryo culture length was three days. If the oocyte pick-up was on Thursday, the minimal culture length was four days.

The latest Cochrane Review, including $27 \mathrm{RCTs}$, detected a higher live birth rate after a blastocyst transfer compared to a cleavage stage embryo transfer [14]. In the present study, we observed similar delivery rates after Day 3 and Day 5 fresh embryo transfers. However, the fresh Day 4 embryos developed to the early blastocyst stage produced the highest live birth rates $(47.4 \%)$ and the lowest miscarriage rates $(6.7 \%)$. Had these embryos been cultured to the blastocyst stage before the transfer, the results of the blastocyst stage transfers might have been improved. The superior results of the early blastocyst transfers on Day 4 could be due to the optimal synchronization of the embryos' developmental stage and the implantation window of the endometrium. It has also been detected that the embryos reaching the blastocyst stage on Day 5 have a higher probability of being euploid than the embryos reaching the blastocyst stage on Day 6. In a study by Kort et al. a higher euploidity rate was detected among early blastocysts (35\%) compared to the morulae $(16 \%)$ on Day 5 , even after controlling for maternal age [15]. It's possible that the embryos reaching the early blastocyst stage on Day 4 have a higher euploidity rate than the slower ones. This, however, remains speculative. 
Day 4 embryo vitrification has been previously described by Zhang et al. who vitrified embryos at the cleavage, morula and blastocyst stages after a biopsy on Day 3 [16]. No difference was detected in the survival rates between the biopsied and non-biopsied morula stage embryos and the biopsied blastocyst stage embryos $(87.5 \%, 92.0 \%$ and $95.7 \%$, respectively). Equal survival rates between Day 4 embryos and blastocysts were also observed in the study at hand. In an earlier study, a markedly lower survival rate was detected among blastocysts compared to the morulae or early blastocysts after vitrification [17]. This effect was dispelled by the artificial shrinkage of the blastocoel cavity after which the survival rate exceeded the survival rate of the morulae or the early blastocysts. The fluid-filled cavity was thought to deserve an extended period of exposure to cryoprotectants, while less osmotic changes occur during cryopreservation at the morula stage.

During the development of an embryo from a zygote to the morula stage, the physiology of the blastomeres converts from a single-celled organism-like physiology to more a somatic cell like physiology due to the first transporting epithelium [18]. Hence, the embryo may be more susceptible to its environment before compaction. An example of the increased resistance of Day 4 embryos towards environmental stress can be found in the increased resistance of the morula to physiological heat shock, compared to the earlier stages of embryo development [19]. This was assumed to be due to the mechanisms which prevent the accumulation of denatured proteins and free radical damage. The development of the morula stage embryo to the blastocyst stage wasn't affected by the heat shock.

The shortcoming of the present study is its retrospective design. The strengths of the study include the fairly substantial study material and reporting the clinically more important live birth rates and miscarriage rates instead of the implantation rates. Our study shows excellent live birth rates and the lowest miscarriage rates associated with both fresh and vitrifiedthawed Day 4 embryo transfers. The mechanism for the success of Day 4 embryos remains speculative. The implementation of the fresh Day 4 embryo transfers and vitrifying the embryos after four days of culture can be considered more often in IVF practice. The Day 4 embryo transfer enables the variation of the possible transfer days and helps to avoid weekend work without impairing the clinical outcomes. Transferring the early blastocyst stage embryos and compacted morulae on Day 4 seems to be particularly effective. A prospective study addressing the clinical outcomes after Day 4 in comparison with the cleavage stage and blastocyst stage transfers with a large study material is needed to verify these results.

\section{References}

1. Edgell TA, Rombauts LJF, Salamonsen LA, et al. Assessing receptivity in the endometrium: The need for a rapid, noninvasive test. Reprod Biomed Online 2013;27:486-496.

2. Ménézo YJ, Bellec V, Zaroukian A, et al. Embryo selection by IVF, co-culture and transfer at the blastocyst stage in case of translocation. Hum Reprod 1997;12:2802-2803.
3. Huisman GJ, Alberda AT, Leerentveld RA, et al. A comparison of in vitro fertilization results after embryo transfer after 2, 3, and 4 days of embryo culture. Fertil Steril 1994;61:970-971.

4. Tao J, Tamis R, Fink K, et al. The neglected morula/ compact stage embryo transfer. Hum Reprod Oxf Engl 2002;17:1513-1518.

5. Pantos K, Makrakis E, Chronopoulou M, et al. Day 4 versus day 3 embryo transfer: A prospective study of clinical outcomes. Fertil Steril 2008;89:573-577.

6. Kang SM, Lee SW, Jeong HJ, et al. Clinical outcomes of elective single morula embryo transfer versus elective single blastocyst embryo transfer in IVF-ET. J Assist Reprod Genet 2012;29:423-428.

7. Lee S-H, Lee H-S, Lim CK, et al. Comparison of the clinical outcomes of day 4 and 5 embryo transfer cycles. Clin Exp Reprod Med 2013;40:122-125.

8. Zander-Fox D, Lane M, Hamilton H. Slow freezing and vitrification of mouse morula and early blastocysts. J Assist Reprod Genet 2013;30:1091-1098.

9. Mohr LR, Trounson A, Freemann L. Deep-freezing and transfer of human embryos. J Vitro Fertil Embryo Transf IVF 1985;2:1-10.

10. Fridström M, Carlström K, Sjöblom P, et al. Effect of prednisolone on serum and follicular fluid androgen concentrations in women with polycystic ovary syndrome undergoing in-vitro fertilization. Hum Reprod Oxf Engl 1999; 14:1440-1444.

11. Gardner DK, Schoolcraft WB. In-vitro culture of human blastocyst. Camforth: Parthenon Press 1999:378-388.

12. Anifandis G, Messini CI, Dafopoulos K, et al. Genes and conditions controlling mammalian pre- and postimplantation embryo development. Curr Genomics 2015;16:32-46.

13. Holschbach V, Weigert J, Dietrich JE, et al. Pregnancy rates of day 4 and day 5 embryos after culture in an integrated time-lapse incubator. Reprod Biol Endocrinol 2017;15:37.

14. Glujovsky D, Farquhar C, Quinteiro Retamar AM, et al. Cleavage stage versus blastocyst stage embryo transfer in assisted reproductive technology. Cochrane Database Syst Rev 2016;6:CD002118.

15. Kort JD, Lathi RB, Brookfield K, et al. Aneuploidy rates and blastocyst formation after biopsy of morulae and early blastocysts on day 5. J Assist Reprod Genet 2015;32:925-930.

16. Zhang X, Trokoudes KM, Pavlides C. Vitrification of biopsied embryos at cleavage, morula and blastocyst stage. Reprod Biomed Online 2009;19:526-531.

17. Vanderzwalmen P, Bertin G, Debauche C, et al. Births after vitrification at morula and blastocyst stages: Effect of artificial reduction of the blastocoelic cavity before vitrification. Hum Reprod Oxf Engl 2002;17:744-751. 
18. Gardner DK, Lane M, Schoolcraft WB. Physiology and culture of the human blastocyst. J Reprod Immunol 2002;55:85-100.
19. Sakatani M, Bonilla L, Dobbs KB, et al. Changes in the transcriptome of morula-stage bovine embryos caused by heat shock: Relationship to developmental acquisition of thermotolerance. Reprod Biol Endocrinol 2013;11:3.

\section{*Correspondence to:}

Noora Kaartinen

Department of Obstetrics and Gynaecology,

Tampere University Hospital,

Teiskontie 35, 33520

Tampere, Finland

Tel: +358331165243

E-mail: noora.kaartinen@pshp.fi 